\title{
Micropulse Transscleral Cyclophotocoagulation: One-year Results and Comparison of two Different Laser Settings in Severe Glaucoma
}

\section{Francesca Chemello}

University of Verona: Universita degli Studi di Verona

Guido Barosco ( $\sim$ guido.barosco@gmail.com )

Universita degli Studi di Verona https://orcid.org/0000-0003-2516-4127

Anna Rodella

University of Verona: Universita degli Studi di Verona

Piero Ceruti

University of Verona: Universita degli Studi di Verona

Roberta Morbio

University of Verona: Universita degli Studi di Verona

Mattia Marcigaglia

University of Verona: Universita degli Studi di Verona

Giorgio Marchini

University of Verona: Universita degli Studi di Verona

\section{Research Article}

Keywords: transscleral laser treatment, micropulse transscleral cyclophotocoagulation, severe glaucoma, refractory glaucoma, laser settings

Posted Date: June 8th, 2021

DOl: https://doi.org/10.21203/rs.3.rs-526291/v1

License: (c) (1) This work is licensed under a Creative Commons Attribution 4.0 International License.

Read Full License 


\section{Abstract}

Purpose: To evaluate the efficacy and safety of micropulse transscleral cyclophotocoagulation (MP-CPC) as a therapeutic option for severe glaucoma and to compare two different durations of treatment.

Methods: Retrospective observational study of 40 eyes of 40 patients affected by different types of glaucoma treated with micropulse transscleral cyclophotocoagulation at $2000 \mathrm{~mW}$ for a duration of either 90 seconds per hemisphere (20 patients) or 80 seconds per hemisphere ( 20 patients) at $31.3 \%$ duty cycle. As main outcomes were evaluated postoperative intraocular pressure (IOP) and topical drugs reduction. Complications of the treatment and a comparative analysis of 2 different durations as secondary outcomes.

Results: MP-CPC was successful in 26 patients (65\%) and achieved a significant IOP reduction of $34.4 \%$ $(p<0.001)$ after 12 months. The mean number of topical antiglaucoma medications has not statistically decreased from baseline $(3.4 \pm 1.0)$ to 12 months $(3.2 \pm 0.8)$. One case of IOP spike $(>35 \mathrm{mmHg})$ and 1 case of cystoid macular oedema were the only complications detected. The comparison between the 90 seconds per hemisphere and the 80 seconds per hemisphere group did not show any statistically significant difference in IOP reduction, topical medication burden and incidence of complications.

Conclusion: MP-CPC demonstrated to be an effective and safe treatment in lowering the IOP. Glaucoma topical medications did not significantly reduce. From our comparative analysis reducing the duration of treatment seems not to affect the outcomes.

\section{Introduction}

Ophthalmologists have always identified in the cycloablation of the ciliary body an effective solution for lowering the intraocular pressure (IOP) reducing the production of aqueous humour [1].

Many different approaches have been used to reach this objective, from the cyclo-diathermy and freezing technique through different types of laser's energy such as Xenon laser, Nd-Yag laser and ruby laser [2].

Continuous-wave transscleral cyclophotocoagulation (CW-CPC) using the diode laser energy has been introduced in 1993 and became the preferred procedure owing to its cost, efficacy and portability [2, 3]. Target of this laser is the pigmented epithelium of the ciliary body: the wavelength employed $(810 \mathrm{~nm})$ is selectively absorbed by these cells, but still, the energy delivered caused a coagulative necrosis of the surrounding tissues as well [4]. In the face of a proven efficacy, the high ratio of serious complications such as macular oedema, vision loss and prolonged hypotony limits its use to refractory glaucoma with low vision potential $[1,5-7]$. All these dangerous complications are secondary to the damages of the surrounding tissues such as the ciliary muscle, the non-pigmented epithelium and the ciliary body stroma [8]. 
The micropulse laser transscleral cyclophotocoagulation (MP-CPC, IRIDEX, CYCLO G6 Glaucoma Laser System, Mountain View, CA) represents the latest news in this panorama. The type of energy and powers are the same of the CW-CPC. The difference stands in the duration in which the energy is provided: the device delivers the energy in a pulsatile wave operating in an "on" and "off" cycle mode with a fixed $31.33 \%$ duty cycle. During the "on" cycle multiple milliseconds repetitive bursts of energy are emitted by the device and absorbed by pigmented tissue, during the "off" cycle non-pigmented tissues and the surrounding stroma have the time to cool off and do not attain the coagulative threshold [9-12]. Beside the primary mechanism based on the reduction of aqueous humour production secondary to the coagulation of ciliary body's pigmented tissue, also an increase of the trabecular outflow as well as the uveo-scleral outflow have been assumed $[1,10,13,14]$. The latest studies suggest a maintenance of the efficacy with a lower incidence of complications compared to the CW-CPC $[8,9]$. The settings on the machine leave the operator free to decide the power of the laser, the duration time of the treatment and the motion of the probe on the patient's conjunctiva.

Literature is still not agreed in terms of efficacy and safety of the procedure as well as duration of treatment and laser parameters to set, influencing the predictability of the treatment $[15,16]$. The first aim of this study is to evaluate the efficacy (considering the amount of reduction of both IOP and antiglaucoma medications) and the safety (considering the rate of complications) of MP-CPC after a follow-up period of 12 months. We also compare 2 different durations of treatment looking for any difference in terms of efficacy and safety of these 2 protocols.

\section{Methods}

This retrospective study involved 40 eyes of 40 adult patients (age $\geq 18$ ) with different types of severe glaucoma. The data were collected reviewing the computerized charts of the first 20 patients consecutively treated with a $90 \mathrm{sec} /$ hemisphere duration and the first 20 patients consecutively treated with an $80 \mathrm{sec} /$ hemisphere duration at the University Eye Clinic of Verona during the first 8 months of usage of the MP-CPC between January and August 2018.

Our Ethical Committee Board approved this study. The research followed the tenets of the declaration of Helsinki. Informed consent to use anonymously the data was obtained from all the subjects. At baseline and at every follow-up visit eye examination, IOP, visual acuity, topical medications, and oral carbonic anhydrase inhibitor (if present) were recorded. In case of failure, re-treatment or other procedures were evaluated from three months from the laser procedure.

These data are referred to our first experience with this new and still not standardized technology and the selection of the patients was linked to our past experience with CW-CPC and with his side effects.

\section{Surgical Technique}


The procedure was delivered by the same two glaucoma specialists (GM, PC) in our operating theatre, all patients received retrobulbar block of 1:1 bupivacaine and mepivacaine before starting the treatment. Methylcellulose gel was applied on the conjunctiva to ensure a better sliding motion of the probe and to properly transmit the energy. The power used was $2000 \mathrm{~mW}$, delivered for either 90 or 80 seconds per hemisphere (superior and inferior) based on the surgeon choice at the day of the treatment, for a total of either 180 or 160 seconds per eye respectively, at $31.3 \%$ of duty cycle. During the procedure the probe was swiped 4 times per hemisphere in the 90 seconds group and 3 times per hemisphere in the 80 seconds group (both superior and inferior), maintaining the perpendicularity with the scleral surface and sparing the 3 and 9 o'clock to avoid the risk of damaging the ciliary nerves. One drop of topical fixed combination of Dexamethasone $1 \mathrm{mg} / \mathrm{ml}$ and Netilmicin $3 \mathrm{mg} / \mathrm{ml}$ were administered to the patients at the end of the treatment. Patients were tapered with topical Dexamethasone $1 \mathrm{mg} / \mathrm{ml}$ for one month after the procedure. All the medications were maintained and eventually modified depending on the need of the patient evaluated at every control visit. Prostaglandin (if present) was suspended for the first 7 days after the procedure, as a standard.

\section{Patients' characteristics}

The study involved 40 eyes of 40 adult patients (age $\geq 18$ ) affected with different types of glaucoma defined as severe stage according to the Hodapp Parish Anderson classification [17]. Demographic and clinical characteristics were recorded at baseline. All patients treated at the time of the procedure had uncontrolled IOP, out of their target values and have no previous history of eye surgery within 6 months from the enrolment or any other cyclodestructive treatment. The duration of treatment received was independent of the preoperatory criteria and up to the surgeon choice. All patients shared clinical and functional indication for the MP-CPC treatment.

\section{Primary Outcomes}

Target IOP was defined as an IOP between 6 and $21 \mathrm{mmHg}$ or a reduction greater than 20\% with or without medications. Treatment success was set as the achievement of the target IOP with or without medications at 1, 3, 6, 12 months follow-up visit. IOP was measured at baseline and at every control visit using the Goldman applanation tonometer (AT $900^{\circledR}$, Haag-Streit International AG, Koeniz, Switzerland). Patients who did not reach their target IOP for $\geq 2$ consecutive visits were scored as failure. Topical drugs reduction from baseline was evaluated at 1 week and 1,3, 6, 12 months follow-up visits.

\section{Secondary Outcomes}

Complications were evaluated at 1, 3, 6, 12 months follow-up visits and included: loss of visual acuity, anterior chamber inflammation, corneal oedema, macular oedema, prolonged hypotony, phthisis bulbi, sympathetic ophthalmia, and any other pathological findings connected to the laser treatment. 
A comparative analysis between the 90 seconds/hemisphere treatment group and the 80 seconds/hemisphere treatment group has been conducted to find any difference in terms of IOP reduction, drugs reduction and rate of complications at 1 week and 1, 3, 6, and 12 months follow- up. Oral carbonic anhydrase inhibitor was the first drug (before topical ones) to be suspended in case of controlled and IOP.

\section{Statistical Analysis}

Data analysis was performed using SPSS for MacOS (version 25.0; SPSS, Inc., Chicago, IL). Normality for data samples was evaluated with the Kolmogorov-Smirnov test. If parametric analysis was possible, the Student's $t$ test for unpaired data was used to compare data between the 80 and 90 seconds/hemisphere groups, whereas for nonparametric analysis the Kruskal-Wallis test was applied to assess the significance of data related to IOP modifications and number of topical drugs needed, post-hoc analysis was conducted performing the Mann-Whitney U-Test and applying the Holm-Bonferroni Correction on the $\mathrm{p}$-value obtained. For all statistical tests, a $p$ value of less than .05 was considered statistically significant.

\section{Results}

The pre-operative patients' characteristics are summarized on Table 1. Seventeen patients (42.5\%) had previously glaucoma surgery (trabeculectomy, deep sclerectomy, canaloplasty and Baerveldt tube implantation); 33 eyes (82.5) had at least one previous eye surgery, included cataract extraction, none had history of cyclodestructive procedures. 


\section{Table 1}

Demographic and Clinical Characteristics of Patients at Baseline

General Group

$21(52 \%)$ male

$19(47 \%)$ female

Age $\quad 65.9( \pm 15.8)(23-88)$

Race/Ethnicity

MD

Lens Status

Prior Eye

Surgery

Types of
Glaucoma

\section{$90 \mathrm{sec} / \mathrm{hemisphere}$}

2 (5\%) Congenital Glaucoma

$1(2.5 \%)$ Neo-Vascular

Glaucoma

1 (2.5\%) Post-Uveitis Glaucoma

1 (2.5\%) Post-PKP Glaucoma group

$9(45 \%)$ male

11 (55\%) female

$66.8( \pm 14.3)(33-83)$

19 White

Caucasian, 1 Latino

18.2 ( \pm 1.8$),(16.2-$

$20.4 \mathrm{~dB})$

10 Phakic, 9

Pseudo-Phakic

15 (79\%) Eyes

12 (63\%) POAG

3 (16\%) PEXG

1 (5\%) ACG

1 (5\%) Silicon-Oil

Glaucoma

\section{$80 \mathrm{sec} / \mathrm{hemisphere}$} group

7 (35\%) male

$13(65 \%)$ female

$62.6( \pm 16.5),(23-88)$

19 White Caucasian, 1

Black African

17.4 ( \pm 1.5$),(15.6-19.3$

$\mathrm{dB})$

13 Phakic, 8 PseudoPhakic

18 (86\%) Eyes

11 (52\%) POAG

3 (14\%) PEXG

3 (14\%) ACG

1 (5\%) Silicon-Oil

Glaucoma

1 (5\%) Congenital

Glaucoma

1 (5\%) Neo-Vascular

Glaucoma

1 (5\%) Post-PKP

Glaucoma

$1(5 \%)$ Congenital

Glaucoma

1 (5\%) Post-Uveitis Glaucoma

$\mathrm{MD}$ = mean deviation; $\mathrm{POAG}$ = primary open angle glaucoma; $\mathrm{PEXG}$ = pseudoexofoliative glaucoma; $A C G=$ angle closure glaucoma; PKP $=$ Penetrating keratoplasty. Age and MD are presented as mean \pm standard deviation and range

The success of MP-CPC treatment was reached by 35 eyes (87.5\%) at 1 month, 32 eyes (80\%) at 3 months, 30 eyes (75\%) eyes at 6 months, and by $26(65 \%)$ at 12 months. After 3 months 8 patients (20\%) were out of their settled range of IOP and needed to be retreated, $4(10 \%)$ underwent successfully a second MP-CPC, 2 (5\%) underwent a Baerveldt tube implantation and 2 (5\%) CW-CPC. After 6 months 2 patients (5\%) did not reach neither their target IOP nor the success and underwent trabeculectomy. After 12 months 4 patients (10\%) had uncontrolled IOP, 1 underwent a microshunt implantation and 3 decided to not receive any other treatment based on a risk-benefit assessment. The mean time of any other 
procedure (MP-CPC, CW-CPC and filtering surgery) was $6.3( \pm 2.2)$ months. Mean IOP \pm standard deviation at baseline was $24.6 \mathrm{mmHg}( \pm 7.2)$. Mean IOP after one treatment was $15.6( \pm 6.9)$; and $16.5( \pm 6.7), 14.5$ $( \pm 5.2), 16.3( \pm 4.5), 16.1( \pm 5.2) \mathrm{mmHg}$, after 1 week, 1 month, 3 months, 6 months and 12 months respectively, with a mean reduction resumed on Figure 1 and Table 2 . The IOP reduction with or without medications was statistically significant $(p<.001)$ at every follow-up visit (Table 2$)$.

\section{Table 2}

Pre- and Post- Treatment Intraocular Pressure and \% of Reduction from Baseline at Different Visits

Mean IOP $(\mathrm{mmHg})$ p-value

$\%$ of Reduction

/

$36 \%$

$33 \%$

$41 \%$

$33 \%$

$34 \%$
I

$<.001$

$<.001$

$<.001$

$<.001$

$<.001$
I

$<.001$

$<.001$

$<.001$

$<.001$

$<.001$
SD

Adjusted-P*

12 Months

16.1

$\mathrm{IOP}=$ intraocular pressure. $\mathrm{SD}=$ standard deviation. $\mathrm{P}^{*}$-Adjusted value via Holm-Bonferroni test to evaluate the statistical significance

Glaucoma medications needed to control IOP lowered from a mean of $3.4( \pm 1.0)$, to $3.20( \pm 0.7), 3.0$ ( \pm 1.3$), 2.9( \pm 1.0), 3.1( \pm 0.8), 3.2( \pm 0.8)$, at baseline and after 1 week, 1 month, 3 months, 6 months, and 12 months respectively (Figure 2). The drugs reduction showed a statistical significance only at 3 months $(p=0.016)$ after surgery. The characteristics of the groups received the 2 different durations of treatment are summarized on Table 1. We did not find any statistically significant difference about the IOP lowering in the two groups (Fig3). The number of medications needed compared in the two groups did not show any statistical relevance (Figure 4).

Rate of complications stood at $5 \%$ ( 2 cases). We had 1 (2.5\%) case of macular oedema on the 80 $\mathrm{sec} /$ hemisphere group with a loss of 2 lines on the Snellen chart from 20/30 to 20/70 and 1 hypertensive IOP spike (38 $\mathrm{mmHg}$ ) on the $90 \mathrm{sec} / \mathrm{hemisphere} \mathrm{group} \mathrm{on} \mathrm{a} \mathrm{refractory} \mathrm{primary} \mathrm{open} \mathrm{angle} \mathrm{glaucoma}$ (POAG), retreated with the MP-CPC reached a stable IOP of $26 \mathrm{mmHg}$ passed from hand motion to light perception and no referred pain. During the follow up 1 patient on the $90 \mathrm{sec} / \mathrm{hemisphere} \mathrm{group} \mathrm{lost} 1$ line on the Snellen chart (from 20/30 to 20/40) due to cataract development not directly ascribable to the laser procedure. No case of prolonged anterior chamber inflammation, phthisis bulbi or corneal (epithelial or endothelial) defect were detected. 
Six (15\%) patients at baseline routinely assumed oral carbonic anhydrase inhibitor, after 12 months follow-up 4 of them belonged to those who needed another surgical/laser treatment, one was prescribed to dismiss his therapy and 1 maintained it.

\section{Discussion}

The advantages of the CW-CPC are usually offset by the very severe disadvantages of this procedure, encouraging to look for a safer and more predictable strategy. Our cohort was made by refractory severe glaucoma, at baseline most of them had already undergone other eye surgical procedures as resumed on Table 1 and would have been good candidates for a CW-CPC, which represented an easy, effective and dangerous option [18-20]. These patients have been treated with the MP-CPC as an alternative to the CW-CPC as a result of the latest evidences of efficacy and safety of this treatment $[8,9,21-23]$. The mean percentage of IOP reduction was 33\% after 1 month, $41 \%$ after 3 months, 33\% after 6 months and $34 \%$ after 12 months (Fig. 1 and Table 2). Our results are concordant with what reported by surgeons who used comparable laser settings and patients' follow-up times [24, 25].

Our success rate did not greatly differ to success rate of Williams et al ( $67 \%$ at 8 months) as well as success rate of Tan et al (73\% at 18 months ) and of the adult group of Lee et al ( $72 \%$ at 12 months); this evidence is corroborated by the percentage values of IOP reduction reported by these three authors, comparable to ours $[22,24,25]$. The reduction on topical medications showed a statistical significance only at month $3(p=0.016)$, though, this reduction has a negligible clinical relevance, changing from a mean $3.4( \pm 1.0)$ to $2.9( \pm 1.0)$. The IOP recorded a higher deflection after 3 months and then slowly increased at month 6 and 12 as recorded on Fig. 2. This observation withstands the mean timing of retreatment in our cohort that was 6.3 months, similar to the mean re-treatment time reported by Aquino et al (6.8 months) [8]. This evidence, if confirmed, induces to think a maximal effect around month 3 based on a balance of aqueous humour reduction, resolution of the inflammation and bio-activation of the drainage pathways, with a downgrading efficacy by the time $[8,12]$.

As far as the complication rate, we had $5 \%$ of complications represented by 1 case of macular oedema and 1 case of IOP spike, successfully managed with a second MP-CPC treatment. This data are closed to the data reported on other studies where similar laser settings have been used $[9,25,26]$. From the charts did not emerged any cased of pathological increase of inflammation secondary to the laser treatment.

The comparison between the group who received 90 seconds/hemisphere and the group who received 80 seconds/hemisphere did not show any statistically significant difference in terms of IOP, medications reduction and rate of complications (Fig. 3 and Fig. 4).

The absence of differences in our two groups could be due to a similar outcome-range of the two durations as supposed from Sanchez et al.[15] Our comparative analysis could suggest that using shorter duration do not greatly affect the efficacy of the treatment. This trend is validated by the results of Sarrafpour et al. who obtained same laser efficacy (30\% IOP lowering) using $2000 \mathrm{~mW}$ for 50 seconds/hemisphere [27]. It is more practical thinking in terms of energy delivered (measured in Joule). 
The physical equation is here expressed:

Energy $=$ Power $x$ Time

Energy $=$ Joule; Power $=$ Watt, Time $=$ seconds

Hence a $2000 \mathrm{~mW}$ treatment delivered in 80 or 90 seconds/hemisphere (180 seconds total) with a duty cycle of $31,3 \%$ corresponds to 100 or 112 Joule of total energy delivered. Likewise, a $2000 \mathrm{~mW}$ treatment delivered in 50 seconds/hemisphere (160 seconds total) with a duty cycle of $31,3 \%$ corresponds to 63 Joule of total energy delivered.

These results leave the field to some speculations: maybe is not a matter of total energy delivered, but of sufficient energy per duty cycle to reach the threshold by the pigmented epithelial cells of the ciliary body, sparing the surrounding stroma. The difficulties putting it into practice and in standardizing the procedure are due to the hidden position of the ciliary body, the interindividual difference in pigmentation that changes the velocity to gain the threshold and the not yet completely defined mechanism of action [28]. Based on our results and on the photophysical response seems reasonable to think about a temperature to reach during a single duty cycle, setting the minimum useful power and exposing the ciliary body to the shorter effective duration.

\section{Conclusion And Study Limitations}

In our series the MP-CPC appears a promising technique combining a satisfying efficacy and safety profile with a good manageability in refractory advanced glaucoma. The difficult aim of the user is to set and maneuver the laser to maximize efficacy minimizing complications. Our comparative analysis suggests that shorter treatment, using the same power setting could maintain the efficacy. More studies should be conducted to better enlighten the right machine settings and to standardize the technique as well as to gain more knowledge about the biological modifications induced by the treatment. A limitation of the study is represented by the variability of the patients' characteristics. Must be noted that to select our subjects we focused the attention on the stage of the disease, regardless of the age of the patients and the type of the glaucoma, since this treatment is mainly reserved to refractory glaucoma, therefore we aimed to investigate it specifically on this category of patients. The absence of randomization proper of a prospective study could be prone to selection bias when it comes to typify different durations of treatment, unfortunately this source of error is intrinsic to the retrospective nature of our investigation. Further prospective studies are needed to confirm our results about the effects related to the duration used.

\section{Declarations}

\section{Funding:}

Not applicable. 


\section{Conflict of interest/Competing interests:}

No conflicting interest exists for any author.

\section{Ethics approval:}

Ethics approval was provided by the Ethical Committee Board of Verona and Rovigo. Written, informed consent was obtained from all the patients. This report does not contain any personal information that could lead to the identification of the patients.

\section{Consent to participate:}

Informed consent was obtained from all the subjects.

\section{Consent for publication:}

Informed consent was obtained from all the subjects.

\section{Availability of data and material:}

Not applicable.

\section{Code availability:}

Not applicable.

\section{Authors' contributions:}

Francesca Chemello: research design, data acquisition, manuscript preparation; Guido Barosco: research design, data acquisition, data analysis, manuscript preparation; Anna Rodella: research design, data acquisition, data analysis, manuscript preparation; Piero Ceruti: research design, data acquisition, manuscript preparation; Roberta Morbio: data acquisition, manuscript preparation; Mattia Marcigaglia: data acquisition, manuscript preparation; Giorgio Marchini: research design, data acquisition, data analysis, manuscript preparation.

\section{References}


1. Kosoko O, Gaasterland DE et al. (1996): Long-term outcome of initial ciliary ablation with contact diode laser transscleral cyclophotocoagulation for severe glaucoma. The Diode Laser Ciliary Ablation Study Group. Ophthalmology 103:1294-1302. doi: 10.1016/s0161-6420(96)30508-3

2. Ndulue JK, Rahmatnejad K et al. (2018) Evolution of Cyclophotocoagulation. J Ophthalmic Vis Res 13:66-61.

3. European Glaucoma Society Terminology and Guidelines for Glaucoma, 4th Edition - Chapter 3: Treatment principles and options Supported by the EGS Foundation: Part 1: Foreword; Introduction; Glossary; Chapter 3 Treatment principles and options. Br. J. Ophthalmol. 2017;101:130-195. doi: 10.4103/jovr.jovr_190_17

4. Schuman JS, Noecker RJ et al. (1991): Energy Levels and Probe Placement in Contact Transscleral Semiconductor Diode Laser Cyclophotocoagulation in Human Cadaver Eyes. Arch Ophthalmol 109, 1534-1538. doi: 10.1001/archopht.1991.01080110070037

5. Nguyen AT, Maslin J, Noecker RJ (2020): Early results of micropulse transscleral cyclophotocoagulation for the treatment of glaucoma. Eur J Ophthalmo/30(4);700-705. doi: $10.1177 / 1120672119839303$

6. Pastor SA, Singh K et al. (2001): Cyclophotocoagulation: a report by the American Academy of Ophthalmology. Ophthalmology 108, 2130-2138.

7. Autrata R, Rehurek J (2003): Long-term results of transscleral cyclophotocoagulation in refractory pediatric glaucoma patients. Ophthalmologica 217, 393-400.

8. Aquino MC, Barton K et al. (2015): Micropulse versus continuous wave transscleral diode cyclophotocoagulation in refractory glaucoma: a randomized exploratory study. Clin Experiment Ophthalmol 43, 40-46. doi: 10.1159/00007306

9. Kuchar S, Moster MR et al. (2016): Treatment outcomes of micropulse transscleral cyclophotocoagulation in advanced glaucoma. Lasers Med Sci 31, 393-396. doi: 10.1007/s10103015-1856-9

10. Fazel F, Bagheri M et al. (2016): Comparison of subthreshold diode laser micropulse therapy versus conventional photocoagulation laser therapy as primary treatment of diabetic macular edema. $J$ Curr Ophthalmol 28, 206-211. doi: 10.1016/j.joco.2016.08.007

11. Brader HS, Young LHY (2016): Subthreshold Diode Micropulse Laser: A Review. Semin Ophthalmol 31, 30- doi: 10.3109/08820538.2015.1114837

12. Amoozgar B, Phan EN et al. (2017): Update on ciliary body laser procedures. Curr Opin Ophthalmol 28, 181-186. doi: 10.1097/ICU.0000000000000351

13. Schubert HD, Agarwala A, Arbizo V (1990): Changes in aqueous outflow after in vitro neodymium: Yttrium aluminum garnet laser cyclophotocoagulation. Investig. Ophthalmol. Vis. Sci. 31, 18341838. 
14. Johnstone M, Padilla S, Wen K (2017): Transcleral laser, ciliary muscle shortening and outflow pathway reorganization. Invest Ophthalmol Vic Sci 58:368.

15. Sanchez FG, Peirano-Bonomi JC, Grippo TM (2018): Micropulse Transscleral Cyclophotocoagulation: A Hypothesis for the Ideal Parameters. Med. Hypothesis, Discov. Innov. Ophthalmol.J. 7, 94-100.

16. Ma A, Yu SWY, Wong JKW (2019): Micropulse laser for the treatment of glaucoma: A literature review. Surv Ophthalmol 64, 486-497. doi: 10.1016/j.survophthal.2019.01.001

17. Hodapp E, Parrish RK II, Anderson DR (1993). In: Mosby Comp (ed.) Clinical Decisions in Glaucoma.St. Louis, MO, USA: The C. V.

18. Walland MJ (2000): Diode laser cyclophotocoagulation: longer term follow up of a standardized treatment protocol. Clin Experiment Ophthalmol 28, 263-267. doi: 10.1046/j.1442-9071.2000.00320

19. Vernon SA, Koppens JM et al. (2006): Diode laser cycloablation in adult glaucoma: long-term results of a standard protocol and review of current literature. Clin Experiment Ophthalmo/34, 411-420. doi:10.1111/j.1442-9071.2006.01241

20. Ramli N, Htoon HM et al. (2012): Risk factors for hypotony after transscleral diode cyclophotocoagulation. J Glaucoma 21, 169-173. doi: 10.1097/IJG.0b013e318207091a

21. Emanuel ME, Grover DS et al. (2017): Micropulse Cyclophotocoagulation: Initial Results in Refractory Glaucoma. J Glaucoma 26, 726-729. doi: 10.1097/IJG.0000000000000715

22. Williams AL, Moster MR et al. (2018): Clinical efficacy and safety profile of micropulse transscleral cyclophotocoagulation in refractory glaucoma. J Glaucoma $27,445-449$.

doi:10.1097/IJG.0000000000000934

23. Toyos MM, Toyos R (2016): Clinical Outcomes of Micropulsed Transcleral Cyclophotocoagulation in Moderate to Severe Glaucoma. J Clin Exp Ophthalmol 07, 7-9.

24. Hui Lee J, Shi Y et al. (2017): Outcome of micropulse laser transscleral cyclophotocoagulation on pediatric versus adult glaucoma patients. J Glaucoma 26, 936-939. doi: 10.1007/s10103-01902934-x

25. Tan AM, Chockalingam $M$ et al. (2010): Micropulse transscleral diode laser cyclophotocoagulation in the treatment of refractory glaucoma. Clin Exp Ophthalmo/38, 266-272. doi: 10.1111/j.14429071.2010.02238.x

26. Gavris MM, Olteanu I et al. (2017): IRIDEX MicroPulse P3: innovative cyclophotocoagulation. Rom J Ophthalmol 61, 107-111. doi: 10.22336/rjo.2017.20

27. Sarrafpour S, Saleh D et al. (2019): Micropulse Transscleral Cyclophotocoagulation. Ophthalmol. Glaucoma 2019;2, 167-171. doi: 10.1016/j.ogla.2019.02.002. 
28. Denton ML, Ahmed EM et al. (2019): Effect of ambient temperature and intracellular pigmentation on photothermal damage rate kinetics. J Biomed Opt 24, 1. doi: 10.1117/1.JBO.24.6.065002

Figures

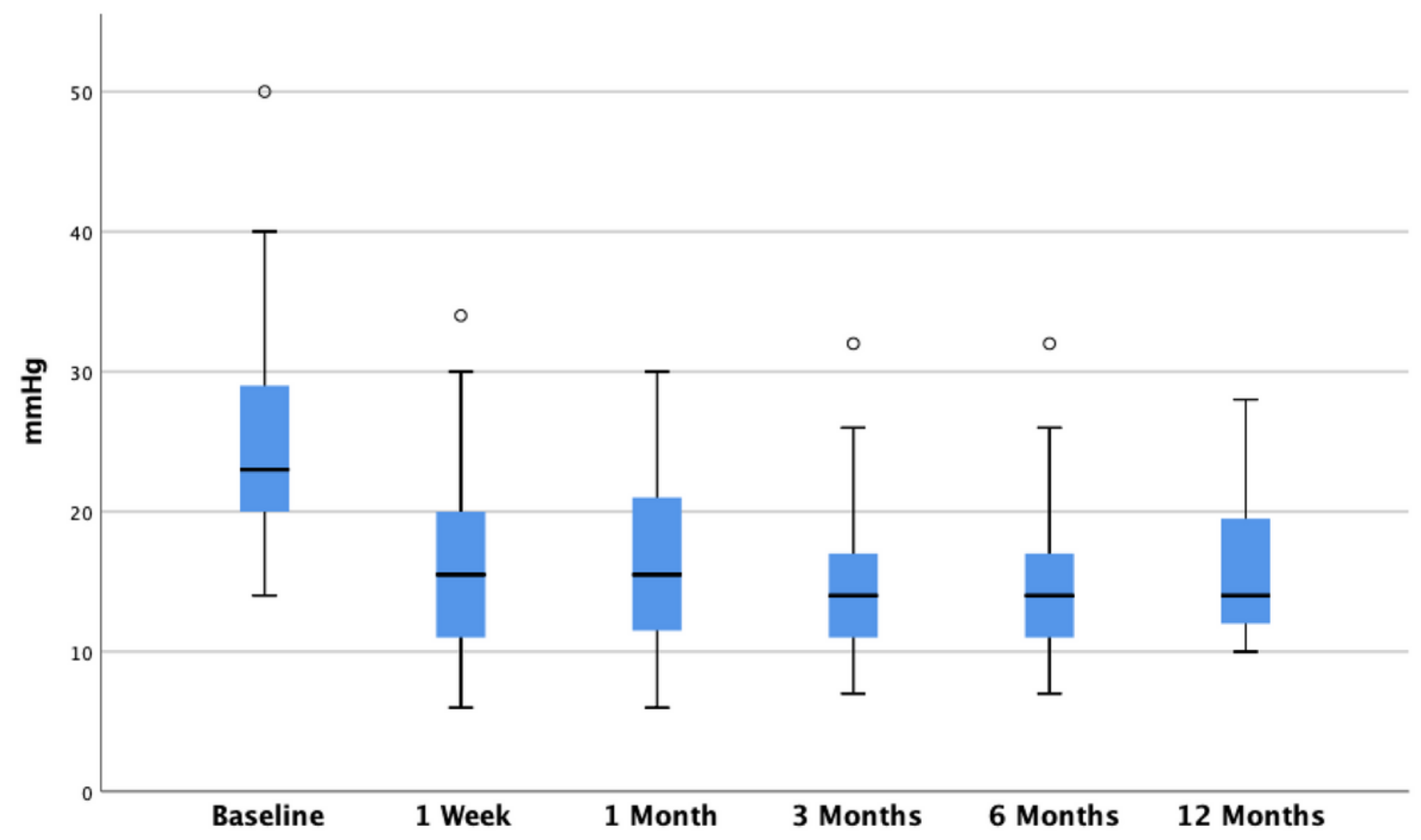

Figure 1

Box plot of IOP values at baseline and at follow-up visits 
5

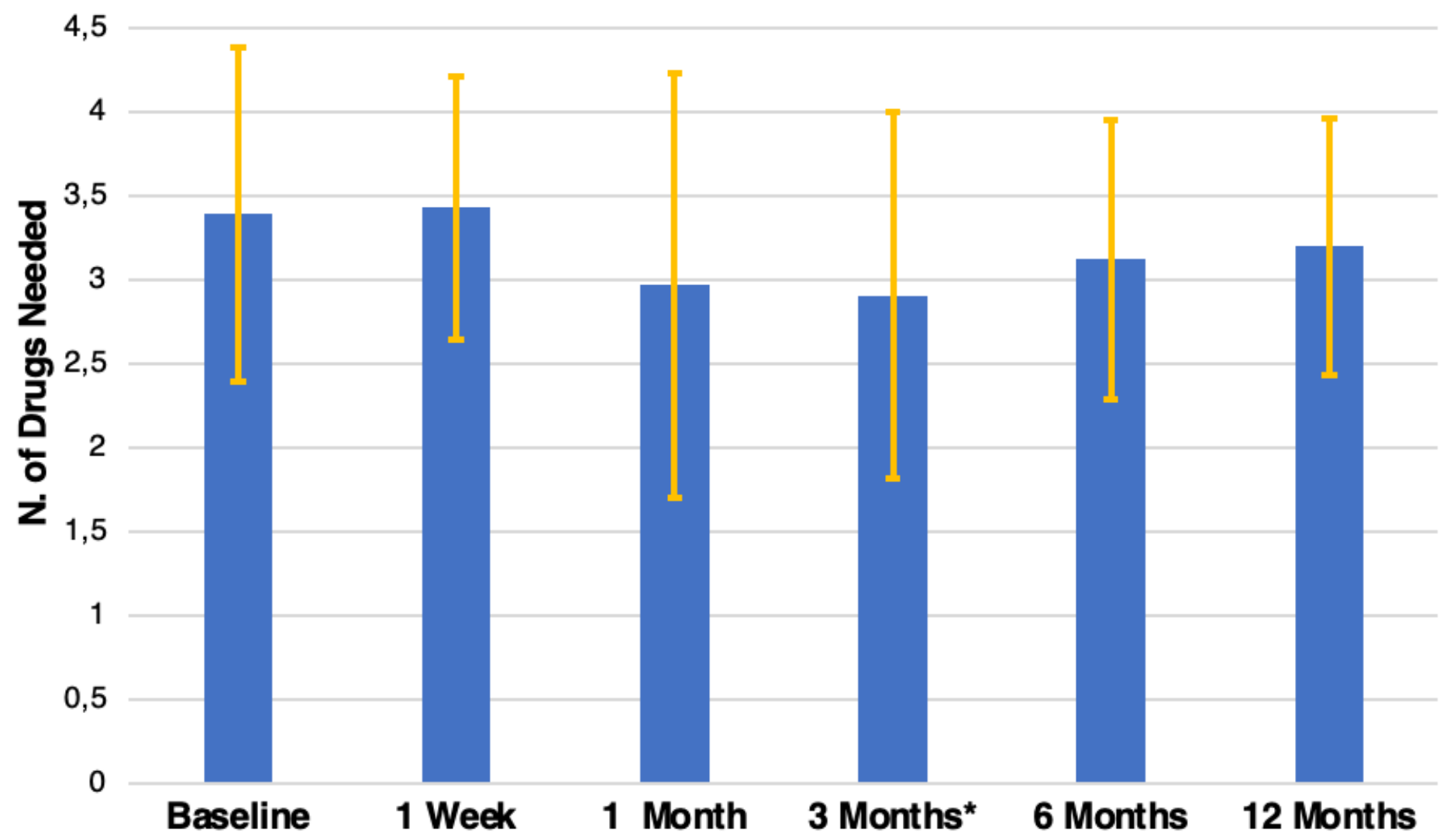

Figure 2

Number of drugs needed to control IOP at baseline and at follow-up visits. Error bars correspond to standard deviation. *3 months drugs reduction was statistically significant 


\section{IOP \\ in 90 vs $80 \mathrm{sec} /$ hemisphere group}

35

30

25

20

15

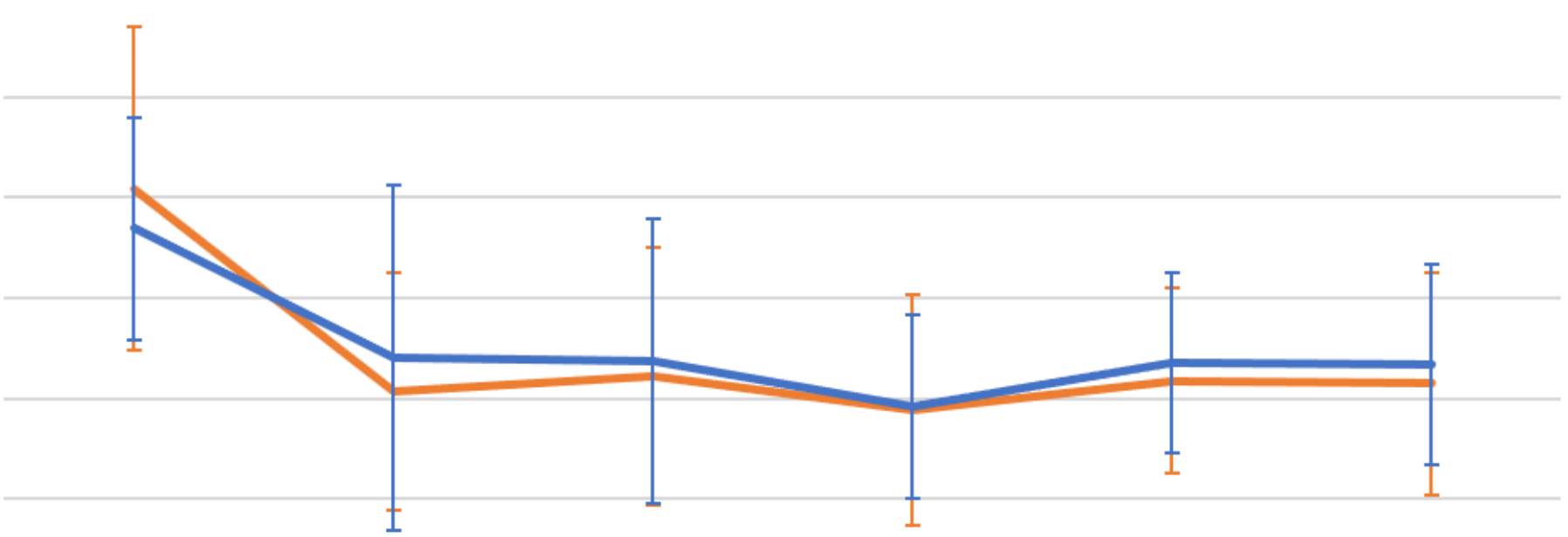

5

0

Baseline

1 Week

1 Month

3 Months

6 Months

12 Months

90 sec/hemisphere group

$80 \mathrm{sec} /$ hemisphere group

Figure 3

Comparison of IOP reduction between the $80 \mathrm{sec} /$ hemisphere group and the $90 \mathrm{sec} /$ hemisphere group at baseline and at follow-up visits. Error bars correspond to standard deviation 


\section{N. of drugs needed in 90 vs $80 \mathrm{sec} / \mathrm{hemisphere} \mathrm{group}$}

6

5

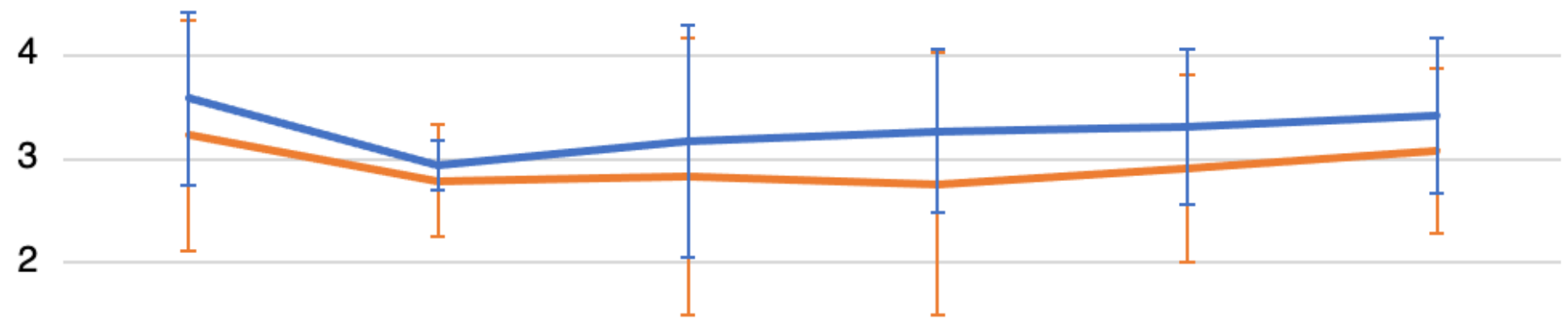

1

0

$\begin{array}{lcccc}\text { Baseline } & 1 \text { Week } & 1 \text { Month } & 3 \text { Months } 6 \text { Months } & 12 \text { Months } \\ & 90 \mathrm{sec} / \text { hemisphere group } & -80 \text { sechemispehre group } & \end{array}$

Figure 4

Comparison of number drugs needed between the $80 \mathrm{sec} /$ hemisphere group and the $90 \mathrm{sec} /$ hemisphere group at baseline and at follow-up visits. Error bars correspond to standard deviation 